\title{
Chemical Ecogenomics-guided Discovery of Phytotoxic $\alpha$-Pyrones from the Fungal Wheat Pathogen Parastagonospora nodorum
}

\author{
Hang Li, Jinyu Hu, ${ }^{\dagger}$ Haochen Wei, ${ }^{\sharp}$ Peter S. Solomon, ${ }^{\sharp}$ Daniel Vuong, ${ }^{\ddagger}$ Ernest Lacey, ${ }^{\ddagger}$ Keith A. Stubbs, ${ }^{\dagger}$ An- \\ drew M. Piggott, $§$ Yit-Heng Chooi ${ }^{*}{ }^{\dagger}$ \\ 'School of Molecular Sciences, The University of Western Australia, Perth, WA 6009, Australia \\ ${ }^{\ddagger}$ Microbial Screening Technologies Pty. Ltd., Smithfield, NSW 2164, Australia. \\ "Research School of Biology, Australian National University, Canberra, ACT 2601, Australia. \\ ${ }^{\S}$ Department of Molecular Sciences, Macquarie University, Sydney, NSW 2109, Australia. \\ Supporting Information Placeholder
}

ABSTRACT: A biosynthetic gene cluster that is significantly upregulated in the fungal wheat pathogen Parastagonospora nodorum during plant infection was reconstructed heterologously in Aspergillus nidulans. This led to the discovery of five new $\alpha$-pyrone polyketides, alternapyrones B-F (2-6). Compounds 5-6, which contain a highly substituted dihydrofuran, exhibited phytotoxicity on wheat seed germination. It is demonstrated that only three enzymes, one highly-reducing polyketide synthase and two multifunctional P450 oxygenases, are needed to synthesize the structurally complex products.

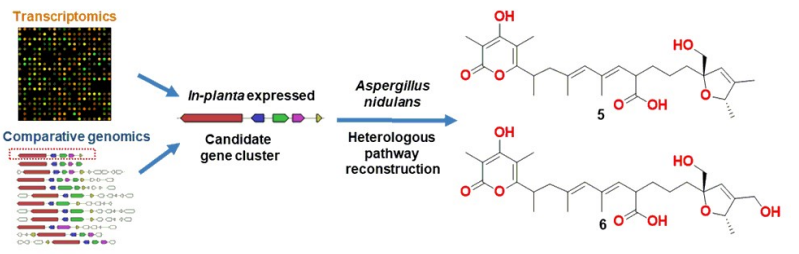

$\alpha$-Pyrone-containing polyketides are an interesting class of natural products that exhibit a wide range of biological activities. ${ }^{1,2}$ Notably, the fungal polyketides citreoviridin, aurovertin and asteltoxin, which all contain an $\alpha$-pyrone linked to highly oxidized heterocyclic systems via a 6-alkenyl chain, are potent F1 ATPase inhibitors (Figure 1). ${ }^{3}$ Additionally, the structurally-related mycotoxin verrucosidin has been reported to be an inhibitor of the mitochondrial electron transport chain complex I. Aurovertins and verrucosidin have also been implicated as potential anticancer therapeutics. ${ }^{5,6}$ Thus, genomics approaches that allow for the discovery of such $\alpha$-pyrone-linked polyketides are highly attractive.

A common feature in the biosynthesis of these $\alpha$-pyrones is that the 6-alkenyl chain with different functionalities synthesized by highly-reducing polyketide synthases (HR-PKSs) is morphed into the various heterocycles by oxidative enzymes. In the case of aurovertin, it has been demonstrated that a single flavin-dependent monooxygenase is involved in the construction of the unusual 2,6dioxabicyclo[3.2.1] octane ring. ${ }^{7}$ It is most likely also the case for citreoviridin and asteltoxin as well. ${ }^{8}$ Alternapyrone (1) is one such 6-alkenylpyrone polyketide, and its dimethylated $\alpha$-pyrone moiety is similar to verucosidin (Figure 1). It was discovered by heterologous expression of a HR-PKS gene PKSN from the tomato and potato pathogen Alternaria solani in Aspergillus oryzae. ${ }^{9}$ However, it is most likely that $\mathbf{1}$ is not the final product in A. solani as the gene cluster encodes an additional three cytochrome P450 and one FAD oxidase gene, which could allow for tailoring of the methylated 6alkenyl chain to generate more complex structures.

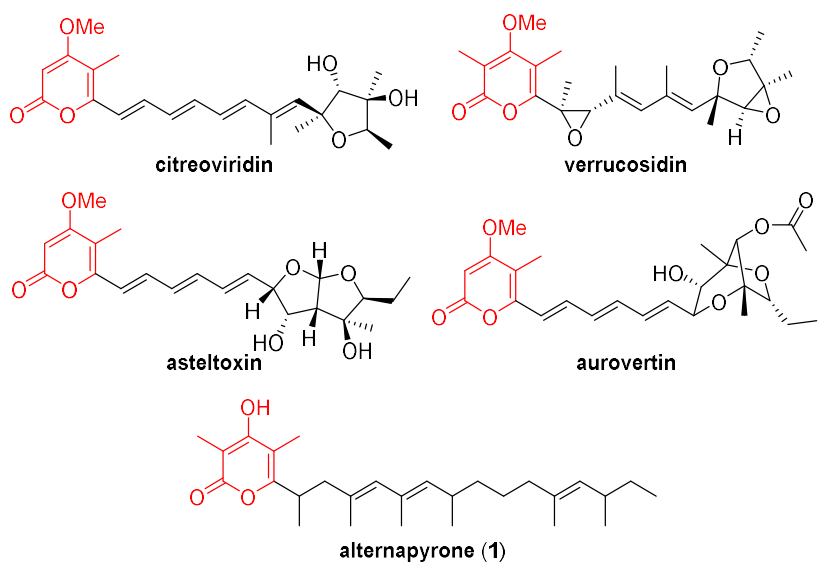

Figure 1. Structures of alternapyrone (1) and representative fungal $\alpha$ pyrone-linked metabolites ( $\alpha$-pyrone highlighted in red). 
Interestingly, a gene cluster in the wheat pathogen Parastagonospora nodorum (SNOG_5791-5797) with significant homology to the A. solani PKSN cluster (Table S2) was shown to be highly upregulated in expression during in planta growth (Figure S1).$^{10}$ Furthermore, homologous gene clusters are also found in many plant pathogens across the Dothideomycete and Sordariomycete classes, including Mycosphaerella populorum (hybrid poplar stem cankers), Pyrenophora tritici-repentis ( $\tan$ spot of wheat), Bipolaris oryzae (brown spot disease in rice), Cochliobolus heterostrophus (corn leaf brown blight) and Colletotrichum fioriniae (bitter rot of apple) (Figure 2A). Significantly, none of the homologous gene clusters in these fungi have been associated with these metabolite products. As part of our ongoing efforts to discover new bioactive metabolites and small molecule virulence mediators from fungi, we set out to characterize the metabolite products from this SN5791-5797 cluster (herein named the alp cluster) as it is expressed during ecological interactions and is conserved across organisms in similar ecological niches, fulfilling the selection criteria for our chemical ecogenomics approach. ${ }^{11}$ Furthermore, the gene cluster holds promise for the discovery of new $\alpha$-pyrones.

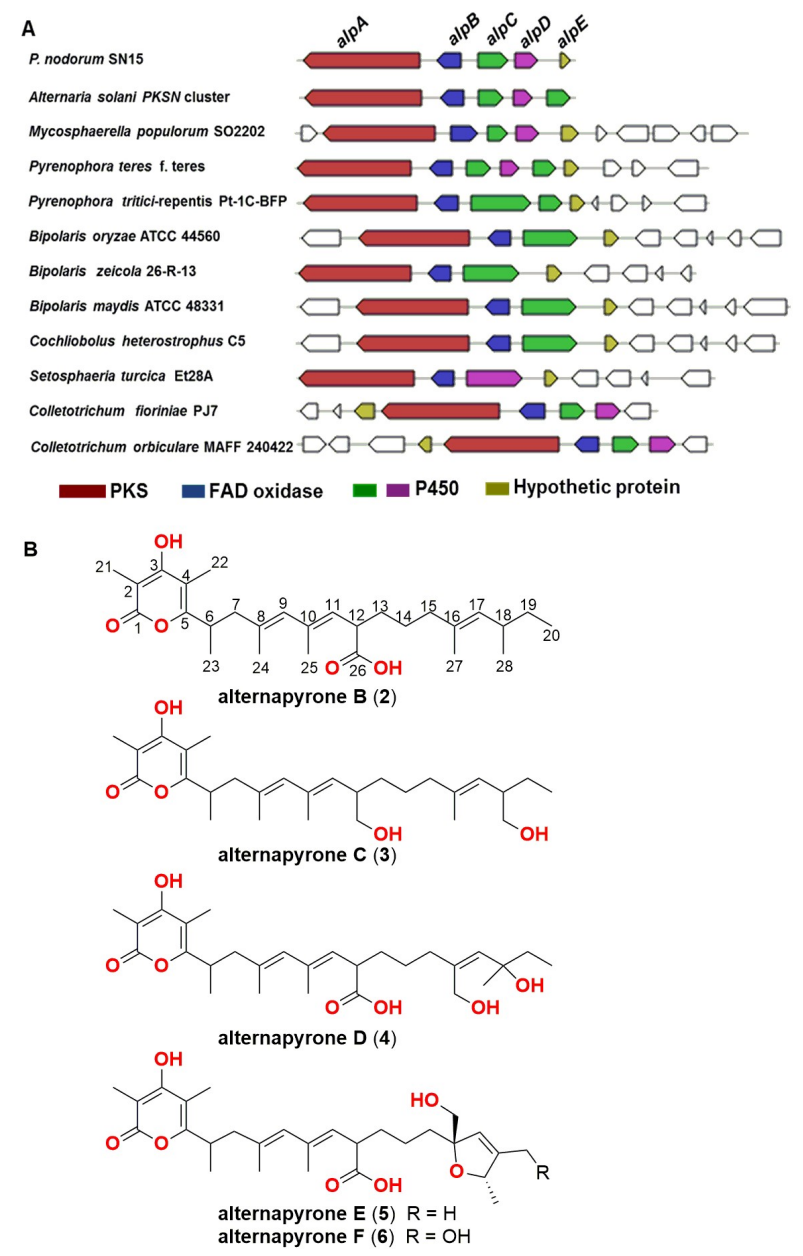

Figure 2. (A) Comparison of gene clusters similar to the alp gene cluster (top). (B) Structures of alternapyrones B-F (2-6) reported in this study.

The alp gene cluster was shown to be expressed at very low levels or is almost silent in in vitro cultures, ${ }^{11}$ and no pathway-specific transcription factor is embedded in this cluster that can be used to activate the expression of silent genes. ${ }^{12}$ Hence, we took a whole pathway reconstruction approach to uncover the metabolite products using a hybrid yeast-fungal artificial chromosome (pYFAC) expression system, which we have derived from a similar episomal expression vector described in previous work. ${ }^{12,13}$ The pYFAC system allows yeast transformation-mediated homologous recombination cloning and episomal expression in A. nidulans. Like the $A$. solani PKSN cluster, the alp cluster encodes two P450 oxygenases (AlpB and AlpC), a FAD-dependent oxidase (AlpD) and a hypothetical protein (AlpE). We first cloned the backbone HR-PKS gene alpA into pYFAC-pyrG under the control of the A. nidulans $\mathrm{P}_{\text {alc }}$ alcohol-inducible promoter to generate the plasmid pYFACCL1. Transformation of $A$. nidulans with pYFAC-CL1 resulted in the production of a new peak $1\left(\mathrm{~m} / z 429[\mathrm{M}+\mathrm{H}]^{+}, 16 \mathrm{mg} / \mathrm{L}\right)$ under induced conditions. Compound $\mathbf{1}$ was subsequently identified as alternapyrone by NMR spectra, ${ }^{9}$ which is not unexpected given that AlpA shares high protein sequence identity to PKSN. Next, we cloned the four putative post-modification genes ( $a l p B, a l p C, a l p D$ and $a l p E$ ) and introduced these genes into pYFAC-ribo under different alcohol-inducible promoters to construct pYFAC-CL2. Then, pYFAC-CL1 and pYFAC-CL2 plasmids were co-introduced into the $A$. nidulans host. Under induced culture conditions, A. nidulans expressing alpABCDE produced five new compounds, $2\left([\mathrm{M}+\mathrm{H}]^{+}\right.$ 459), $3\left([\mathrm{M}+\mathrm{H}]^{+} 461\right), 4\left(\left[\mathrm{M}-\mathrm{H}_{2} \mathrm{O}+\mathrm{H}\right]^{+} 473\right), \mathbf{5}\left([\mathrm{M}+\mathrm{H}]^{+} 489\right)$ and $6\left([\mathrm{M}+\mathrm{H}]^{+} 505\right)$, with 5 as the most abundant product. No trace of 1 was observed and a subsequent scaled-up culture $(4 \mathrm{~L})$ and HPLC purification yielded $2(10 \mathrm{mg}), 3(2.4 \mathrm{mg}), \mathbf{4}(15 \mathrm{mg}), \mathbf{5}$ (48 $\mathrm{mg})$ and $6(14 \mathrm{mg})$.

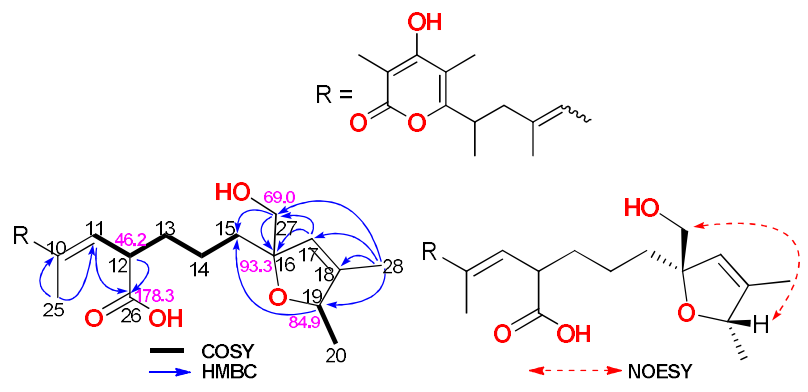

Figure 3. Key 2D NMR correlations of $\mathbf{5}$. Key ${ }^{13} \mathrm{C}$ NMR chemical shifts are shown in pink.

Based on HR-ESI-MS and extensive 1D and 2D NMR analyses, we elucidated the structures of $\mathbf{2 - 6}$, which we named alternapyrones B-F, respectively (Figure $2 \mathrm{~B}$ ). Compounds 2 and 3 were shown to be the 26-carboxy and 26,28-dihydroxy derivatives of $\mathbf{1}$ respectively. Similarly, 4 was shown to be the 18,27-dihydroxy derivative of 2 , possessing a tertiary alcohol at C-18 (Tables S8 and S11). 2D NMR analysis of 5 revealed the C-16/17 double bond had shifted to C-17/18 and that C-19 and C-27 were oxygenated, with a free hydroxy group on C-27 and C-19 connected to C-16 via an ether linkage, thus forming a 2,5-dihydrofuran (Figure 3). This structure was supported by a long-range $\mathrm{HMBC}$ correlation from $\mathrm{H}-19$ to $\mathrm{C}-15$. The relative configuration of the dihydrofuran ring was determined by $1 \mathrm{D}$ NOESY, which revealed a correlation between $\mathrm{H}-19$ and $\mathrm{H}_{2}-27$ (Figure 3). Compound 6 differed from 5 only by the presence of an additional hydroxy group on C-28. The experimental ${ }^{13} \mathrm{C}$ NMR shifts for 4-6 matched closely with theoretical values calculated using density functional theory (DFT; defTZVP, B3-LYP) (Tables S11-S13). 
To characterize the biosynthetic logic of alternapyrones 2-6, different gene combinations were constructed using the pYFAC expression system. A. nidulans co-expressing $a l p A$ and $a l p D$ resulted in production of the carboxylic acid $\mathbf{2}$ instead of $\mathbf{1}$, while coexpression of any other tailoring genes with alpA only resulted in production of 1 (Figure 4). This clearly demonstrated that alpD oxidizes the C-26 methyl group of $\mathbf{1}$ to a carboxyl group, and suggests that this is likely to be the first post-PKS tailoring step after the formation of $\mathbf{1}$. We next focused on the subsequent catalytic steps by co-expressing individual remaining tailoring genes with alp $A / D$. Interestingly, only co-expression with $a l p C$, another $\mathrm{P} 450$ oxygenase encoded in the gene cluster, together with $\operatorname{alp} A / D$, was sufficient to produce 2-6 in the $A$. nidulans host (Figure 4). Introduction of either $a l p B$ or $a l p E$ to the alpA/D constructs did not result in strains that produced any further new metabolites, suggesting that $a l p D$ and $a l p C$ are sufficient to convert $\mathbf{1}$ to 2-6.

The elucidated structures of 2-6 suggest that the biosynthetic pathway toward these new alternapyrones is highly branched (Scheme 1). Based on the genes that were shown to be involved in the synthesis and the established biosynthetic logic, we propose that the HR-PKS AlpA synthesizes the alternapyrone polyketide backbone and releases it via spontaneous lactonization when a tri$\beta$-keto intermediate is formed, as shown previously. ${ }^{14}$ Then, AlpD oxidizes the C-26 methyl group via a triple oxidation to afford the carboxylic acid 2. Such P450 oxygenases that catalyze the formation of a carboxylate group from a methyl group via consecutive oxidations are precedented in fungi. ${ }^{15}$ Interestingly in $\mathbf{3}$, oxidation at $\mathrm{C}$ 26 stops at the intermediate primary alcohol and another primary alcohol is installed on C-28, when alpC was added to A. nidulans expressing alp $A / D$. This suggests that AlpC oxidizes C- 28 before AlpD completely converts $\mathbf{1}$ to $\mathbf{2}$, and this oxidation of C-28 prohibits AlpD from oxidizing further the primary alcohol on C-26. It is also possible that $\mathbf{3}$ is a shunt intermediate in the culture.

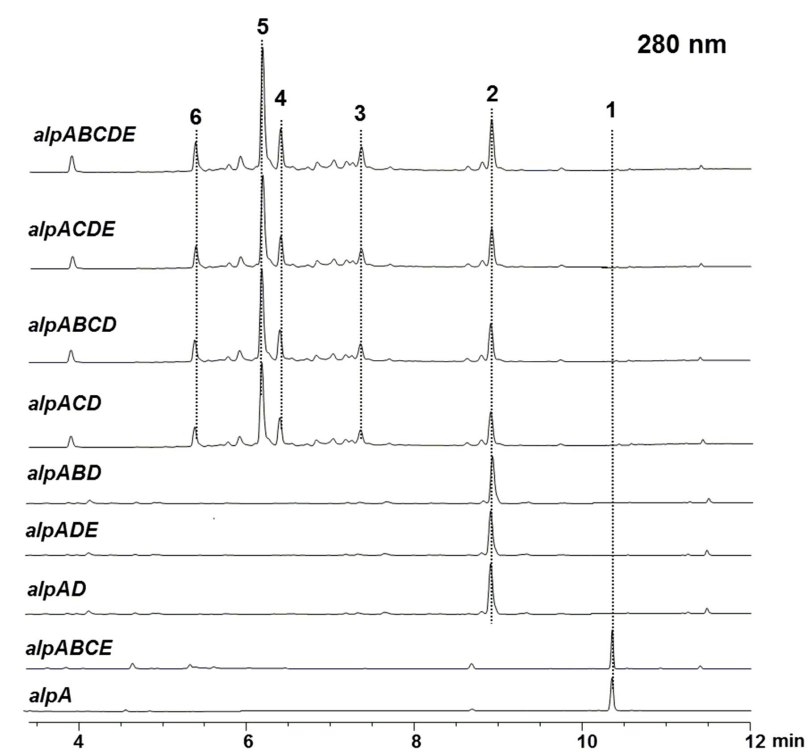

Figure 4. HPLC traces $(280 \mathrm{~nm})$ showing metabolite profile from $A$. nidulans transformants.

From 2, AlpC hydroxylates C-27 and then $\mathrm{t}$ pathway branches out to 4 by hydroxylation at C-18 (Scheme 1). Notably, the $18-\mathrm{OH}$ on 4 is a tertiary alcohol. Some P450 oxygenases are known to cata- lyze tertiary alcohol formation, and they are valuable biocatalysts. ${ }^{16}$

${ }^{17}$ Compounds $\mathbf{5}$ and $\mathbf{6}$ with a dihydrofuran moiety are noted to have a double bond that is shifted from C-16/17 to C-17/18. We reasoned this could be the result of dehydration of $27-\mathrm{OH}$, which is also found in 4, resulting in the formation of a C-16/27 terminal olefin and shifting of the existing double bond to C-17/18. Epoxidation of the olefin by AlpC followed by hydroxylation at C-19 sets the intermediate up for a 5-exo-tet cyclization to afford 5. Finally, hydroxylation at C-28 results in $\mathbf{6}$. Epoxide-opening cascades are well-known to be involved in the formation of heterocycles. For example, in the case of aurovertin, two consecutive epoxidations, ring openings and rearrangements results in a tetrahydrofuran intermediate, which is followed by the formation of the 2,6dioxabicyclo[3.2.1] octane ring. ${ }^{7}$ Even though multifunctional P450 oxygenases are now well-known, e.g. in fumagillin ${ }^{18}$ and terretonin $\mathrm{H}^{19}$ biosynthesis in fungi, they continue to be a rich source of new catalytic abilities. The results together suggest that the P450 AlpC is most likely involved in the hydroxylation, terminal olefin formation, epoxidation and cyclization steps leading to the formation of $\mathbf{5}$ as it is the major product of $A$. nidulans alpACD. Nonetheless, we cannot completely rule out the involvement of endogenous host enzymes until further in vitro enzymatic study is performed.

\section{Scheme 1. Proposed biosynthetic pathway for 2-6.}

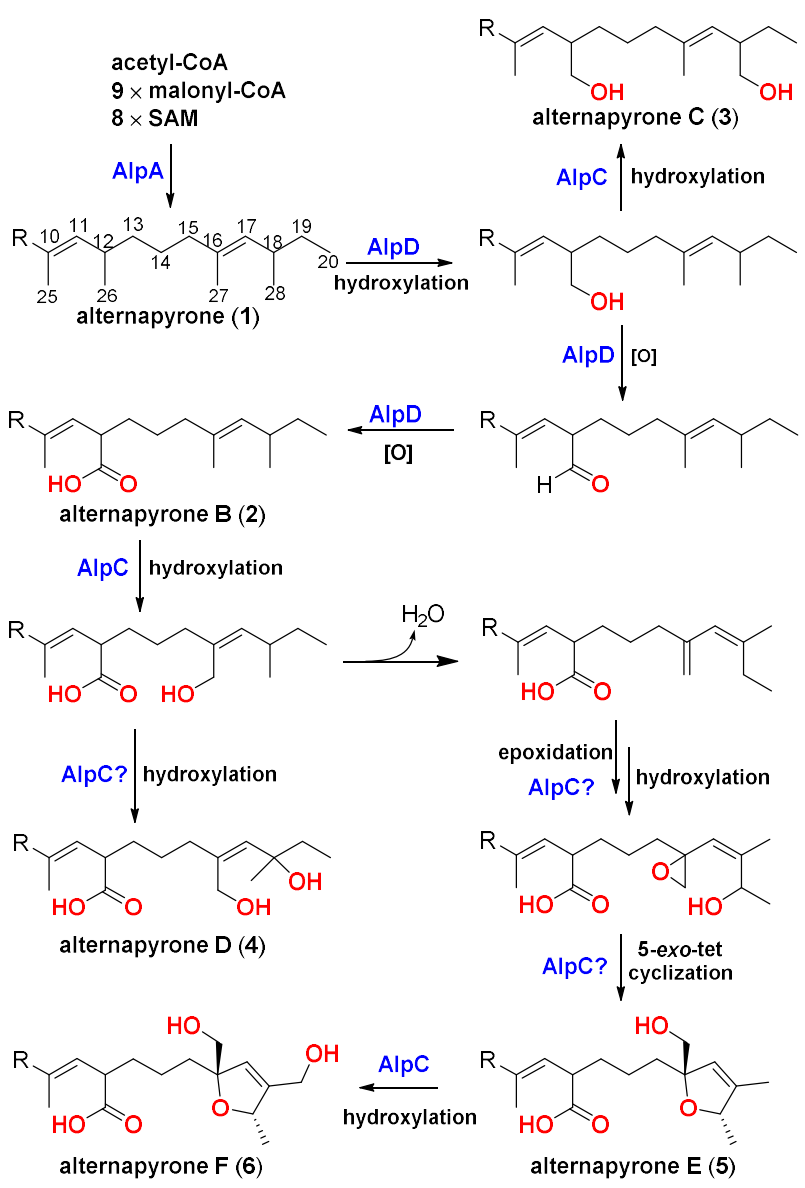

Equipped with 1-6, we next tested if these compounds exhibited any phytotoxic activity. No necrosis was observed on wheat leaves infiltrated with up to $200 \mu \mathrm{g} / \mathrm{mL}$ of 1-6. However, seed germination assays showed that alternapyrones D-F (4-6) exhibited wheat anti-germination activities, most notably alternapyrone $\mathrm{F}(\mathbf{6})$, 
which completely inhibited wheat germination at $100 \mu \mathrm{g} / \mathrm{mL}$ (Figures 5 and S4). Concurrently, we also tested 1-6 for other bioactivities (antibacterial, antifungal, antiparasitic and antitumor) (Table 1). Alternapyrone (1) displayed cytotoxic activity against murine myeloma $(\mathrm{MIC}=3.1 \mu \mathrm{g} / \mathrm{mL}$ ) but was 8 -fold less active against neonatal foreskin fibroblasts non-tumor cells $(\mathrm{MIC}=25 \mu \mathrm{g} / \mathrm{mL})$, suggesting the cytotoxicity has some specificity against tumor cells. Surprisingly, the highly oxidized and heterocycle-containing 5-6, which exhibited higher anti-germinative activity, displayed no bioactivity at all in the bioassays used, suggesting the mode of action of the cytotoxicity and phytotoxicity is different.

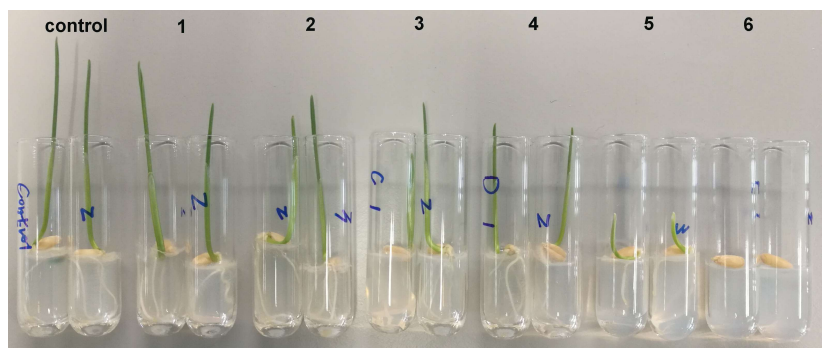

Figure 5. Effects of alternapyrones 1-6 on wheat seed germination at $100 \mathrm{ppm}$ on Day 5. Experiments were performed in duplicate.

Table 1. Bioactivities of alternapyrones 1-6.

\begin{tabular}{ccccccc} 
Compd & \multicolumn{7}{c}{ MIC $(\mu \mathrm{g} / \mathrm{mL})$} \\
& $B s$ & $E c$ & $C a$ & $G d$ & $M m$ & $N f f$ \\
$\mathbf{1}$ & 100 & $>10$ & $>200$ & 50 & 3 & 25 \\
$\mathbf{2}$ & 12.5 & $>10$ & $>200$ & 25 & 13 & 100 \\
$\mathbf{3}$ & 25 & $>25$ & $>50$ & $>25$ & $>25$ & $>25$ \\
$\mathbf{4}$ & $>10$ & $>10$ & $>200$ & $>100$ & 100 & $>100$ \\
$\mathbf{5}$ & $>10$ & $>10$ & $>200$ & $>100$ & 50 & 100 \\
$\mathbf{6}$ & $>10$ & $>10$ & $>200$ & $>100$ & $>100$ & $>100$
\end{tabular}

Note: Bs, Bacillus subtilis; Ec, Escherichia coli; Ca, Candida albicans; $\mathrm{Gd}$, Giardia duodenalis; $\mathrm{Mm}$, murine myeloma; Nff, neonatal foreskin fibroblasts.

Given that the alp cluster is highly upregulated during in planta growth compared to in vitro and that 5-6 exhibited phytotoxicity on wheat seed germination, we tested whether they play any role in virulence. We constructed $P$. nodorum $\triangle a l p A$ mutants and performed whole wheat spray assays with these mutants in comparison to wild-type as described previously. ${ }^{12}$ However, no significant difference in disease symptoms between wheat leaves inoculated with $\triangle a l p A$ mutants and controls (wild-type) was observed (Figure S3). Together these results suggest that the alp pathway products play no important role in lesion development of wheat, but may interfere with other cellular or developmental pathways not be fully captured via this simple seedling infection assay. Furthermore, the effects of $\triangle a l p A$ could be masked by other virulence factors from $P$. nodorum.

In conclusion, we have identified a new group of bioactive $\alpha$ pyrones including 5 and $\mathbf{6}$, which are linked to highly-oxidized heterocyclic systems. This study, along with the previous discovery of the mellein ${ }^{20}$ and elsinochrome ${ }^{12}$ pathways in P. nodorum, demonstrates that such ecology-guided genome mining approaches are a promising strategy to undercover bioactive metabolites relevant in an ecological context. Homology to the previously characterized $A$. solani PKSN provides a hint to the potential structures of the products of the alp cluster. Compounds 2-6 differ from the structurally- related $\alpha$-pyrones aurovertin, citreoviridin and asteltoxin in that the 6-alkenyl side chain are not polyene-like and have a more complex methylation pattern. Bioinformatics analysis revealed that the alp/alt gene cluster is conserved across many plant pathogens, but there appears to be variation in the number and type of tailoring oxidative enzymes (Figure 2A). Thus, these homologous clusters provide a source of discovery for novel $\alpha$-pyrone compounds, which may be linked to different heterocyclic systems. The successful heterologous expression of the pathway using the A. nidulans host further sets the foundation for combinatorial biosynthesis of this subclass of 6-alkenylpyrone compounds.

\section{AUTHOR INFORMATION}

\section{Corresponding Author}

*Email: yithengchooi@uwa.edu.au

Notes

The authors declare no competing financial interest.

\section{ACKNOWLEDGMENT}

This study was supported by an Australian Research Council (ARC) Discovery Project grant (DP170100228). Li H. and Hu J. are supported by an Australian Government International Postgraduate Research Scholarship. Y-HC, KAS and AMP are ARC Future Fellows. The authors thank Dr Gareth Nealon (UWA) for assistance with 1D NOESY NMR experiments. NMR and HR-MS were performed at the UWA Centre for Microscopy, Characterisation \& Analysis (CMCA). DFT calculations were performed with the assistance of resources from the Australian National Computational Infrastructure (NCI).

\section{REFERENCES}

1. Dickinson, J. M. Nat. Prod. Rep. 1993, 10, 71.

2. McGlacken, G. P.; Fairlamb, I. J. S. Nat. Prod. Rep. 2005, 22,

369.

3. Hong, S.; Pedersen, P. L. Microbiol. Mol. Biol. Rev. 2008, 72,

590.

4. Thomas, S.; Sharma, N.; Gonzalez, R.; Pao, P.-W.; Hofman, F. M.; Chen, T. C.; Louie, S. G.; Pirrung, M. C.; Schönthal, A. H. PLoS One 2013, 8, e65695.

5. Huang, T.-C.; Chang, H.-Y.; Hsu, C.-H.; Kuo, W.-H.; Chang, K.-J.; Juan, H.-F. J. Proteome Res. 2008, 7, 1433.

6. Park, H.-R.; Ryoo, I.-J.; Choo, S.-J.; Hwang, J.-H.; Kim, J.-Y.; Cha, M.-R.; Shin-Ya, K.; Yoo, I.-D. Toxicology 2007, 229, 253.

7. Mao, X.-M.; Zhan, Z.-J.; Grayson, M. N.; Tang, M.-C.; Xu, W.; Li, Y.-Q.; Yin, W.-B.; Lin, H.-C.; Chooi, Y.-H.; Houk, K. N.; Tang, Y. J. Am. Chem. Soc. 2015, 137, 11904.

8. Lin, T.-S.; Chiang, Y.-M.; Wang, C. C. C. Org. Lett. 2016, 18, 1366.

9. Fujii, I.; Yoshida, N.; Shimomaki, S.; Oikawa, H.; Ebizuka, Y. Chem. Biol. 2005, 12, 1301.

10. Ipcho, S. V. S.; Hane, J. K.; Antoni, E. A.; Ahren, D. A. G.; Henrissat, B.; Friesen, T. L.; Solomon, P. S.; Oliver, R. P. Mol. Plant Pathol. 2012, 13, 531.

11. Chooi, Y.-H.; Solomon, P. S. Fron. Microbiol. 2014, 5, 640.

12. Chooi, Y.-H.; Zhang, G.; Hu, J.; Muria-Gonzalez, M.J.; Tran, P. N.; Pettitt, A.; Maier, A. G.; Barrow, R. A.; Solomon, P. S. Environ. Microbiol. 2017, 19, 1975.

13. Sato, M.; Yagishita, F.; Mino, T.; Uchiyama, N.; Patel, A.; Chooi, Y.-H.; Goda, Y.; Xu, W.; Noguchi, H.; Yamamoto, T.; Hotta, K.; Houk, K. N.; Tang, Y.; Watanabe, K. ChemBioChem 2015, 16, 2294.

14. Chooi, Y.-H.; Tang, Y.J. Org. Chem. 2012, 77, 9933.

15. Van de Bittner, K. C.; Nicholson, M. J.; Bustamante, L. Y.; Kessans, S. A.; Ram, A.; van Dolleweerd, C. J.; Scott, B.; Parker, E. J. J. Am. Chem. Soc. 2018, 140, 582. 
16. O'Reilly, E.; Kohler, V.; Flitsch, S. L.; Turner, N. J. Chem.

Commun. 2011, 47, 2490.

17. Kirsten, S.; Matthias, K.; Stephan, L. Biotechnol. Bioeng. 2010 $106,699$.

18. Lin, H.-C.; Tsunematsu, Y.; Dhingra, S.; Xu, W.; Fukutomi, M.; Chooi, Y.-H.; Cane, D. E.; Calvo, A. M.; Watanabe, K.; Tang, Y. J. Am. Chem. Soc. 2014, 136, 4426.

19. Matsuda, Y.; Iwabuchi, T.; Wakimoto, T.; Awakawa, T.; Abe, I. J. Am. Chem. Soc. 2015, 137, 3393.

20. Chooi, Y.-H.; Krill, C.; Barrow, R. A.; Chen, S.; Trengove, R.; Oliver, R. P.; Solomon, P. S. Appl. Environ. Microbiol. 2015, 81, 177. 